\title{
Measurements of $L$-shell x-ray production cross sections of W, Pt, and Au by 10-30-keV electrons
}

\author{
C. S. Campos and M. A. Z. Vasconcellos \\ Instituto de Física, Universidade Federal do Rio Grande do Sul (UFRGS), 91501-970 Porto Alegre, RS, Brazil \\ X. Llovet* \\ Serveis Cientificotècnics, Universitat de Barcelona, Lluís Solé i Sabarís 1-3, 08028 Barcelona, Spain \\ F. Salvat \\ Facultat de Física (ECM), Universitat de Barcelona, Diagonal 647, 08028 Barcelona, Spain
}

(Received 19 March 2002; published 30 July 2002)

\begin{abstract}
We present results from measurements of $L \alpha$ x-ray production cross sections of the elements $\mathrm{W}, \mathrm{Pt}$, and $\mathrm{Au}$ by impact of electrons with energies in the range 10-30 keV. The cross sections were obtained by measuring $L \alpha$ x-ray intensities emitted from very thin films of the studied elements deposited on thick carbon substrates. The directional and energy spreading of the electron beam within the active film and the x-ray enhancement due to electron backscattering from the substrate were accounted for by means of Monte Carlo simulation. Recorded x-ray intensities were converted to absolute x-ray production cross sections by using two different methods; the first employs measured values of the sample thickness and the number of incident electrons and estimated detector efficiencies; the second is based on a comparison between measured and calculated bremsstrahlung intensities. Experimental data are compared with the results of simple analytical formulas of common use in practical electron probe microanalysis, with calculated cross sections obtained from the distorted-wave Born approximation and with other experimental data available in the literature.
\end{abstract}

DOI: $10.1103 /$ PhysRevA.66.012719

PACS number(s): $34.80 . \mathrm{Dp}$

\section{INTRODUCTION}

Accurate cross sections for inner-shell ionization by electron impact are required for multiple applications, particularly for electron probe microanalysis (EPMA) and Augerelectron spectroscopy. In spite of this need, a systematic method for calculating accurate ionization cross sections from first-principles remains to be found. The usual practice consists of using semiempirical formulas, which have limited ranges of validity and accuracy; too frequently different formulas lead to significantly different results. Calculations within the plane-wave first Born approximation provide reliable results for high-energy electrons [1]; however, this approach is not satisfactory near the ionization threshold. A more appropriate theoretical tool is provided by the distorted-wave Born approximation (DWBA), even though the calculations are extremely time consuming and difficult to validate [2].

In the energy range of interest in EPMA, say 1-50 keV, experimental measurements of inner-shell ionization cross sections deal mostly with $K$ shells; cross-section data for $L$ and $M$ shells are very scarce. Absolute $L$-shell ionization cross sections have only been reported for a few elements such as $\mathrm{Ar}[3,4], \mathrm{Kr}$ [5], $\mathrm{Xe}$ [5,6], Au [7-10], and W [11] (see also Refs. [12-14]). Moreover, available experimental data are affected by large uncertainties and important discrepancies are found when comparing data from different authors $[15,16]$. As a consequence, it is difficult to assess the reliability of cross sections calculated with the DWBA or

\footnotetext{
*Author to whom correspondence should be addressed. Email address: xavier@giga.sct.ub.es
}

other approximation. The situation is even worse for $M$ shells, for which experimental data are extremely rare. New, accurate experimental measurements of $L$ - and $M$-shell ionization cross sections by $\mathrm{keV}$ electron impact are therefore urgently needed.

Inner-shell ionization cross sections can be determined by measuring the intensity of characteristic x-rays emitted from self-supporting thin films of the considered elements, bombarded by an electron beam [17]. The determination of absolute values of the cross section, however, poses numerous difficulties (see e.g., Ref. [18]). In the case of $L$ and $M$ shells, adding to these difficulties is the fact that vacancies in a given subshell can be produced not only by electron impact but also by nonradiative (Coster-Kronig) transitions between the subshells. As a consequence, the intensity of a given $\mathrm{x}$-ray line depends on the ionization cross sections of all the subshells, weighted by the corresponding Coster-Kronig coefficients. Consequently, to determine subshell ionization cross sections we have to measure the intensities of a number of x-ray lines, some of which may not be clearly resolved or may have very low intensities. Moreover, Coster-Kronig coefficients are generally affected by large uncertainties, which would propagate to the derived ionization cross sections. Considering these difficulties, it is advisable to report the cross section for $\mathrm{x}$-ray production, usually for the most intense lines, rather than the cross section for inner-shell ionization. By proceeding in this way, Coster-Kronig coefficients, fluorescence yields, and fractional emission rates do not affect the reported experimental data. It should also be noted that for many applications, including EPMA, the goal is to calculate $\mathrm{x}$-ray intensities from irradiated samples, which can be obtained from knowledge of the cross section for $\mathrm{x}$-ray production. 
In this work, we report on experimental measurements of $L \alpha$ x-ray production cross sections for the elements $\mathrm{W}$ ( $Z$ =74), Pt $(Z=78)$, and $\mathrm{Au}(Z=79)$, for incident electron energies $10-30 \mathrm{keV}$. Cross sections were obtained by measuring $L \alpha$ x-ray intensities emitted from very thin films of the studied elements, which were deposited on carbon substrates. Measurements were performed with an electron microprobe, by using both a $\mathrm{Si}(\mathrm{Li})$ detector and a crystal spectrometer. The effects of spatial and energy spreading of the electron beam within the active film and the x-ray enhancement due to electron backscattering from the substrate were corrected by using Monte Carlo (MC) simulation results generated by considering the particular geometry of each sample. $L \alpha$ x-ray intensities were converted to $L \alpha$ x-ray production cross sections by two different methods (i) by using measured values of the sample thickness and the number of incident electrons and estimated detector efficiencies and (ii) by using measured and calculated bremsstrahlung intensities. Results are compared with simple analytical formulas of common use in EPMA and with calculated cross sections obtained from the DWBA. To make this comparison possible, theoretical ionization subshell cross sections have been converted to x-ray production cross sections by using relaxation data available from the literature. Our experimental results are also compared with measurements of other authors.

\section{EXPERIMENTAL PROCEDURE}

The apparatus used for electron bombardment and for the detection of $\mathrm{x}$-ray spectra from the specimen was a CAMECA SX-50 electron microprobe (Microprobe Laboratory, Universidade Federal do Rio Grande do Sul). In this instrument X-ray spectra can be measured simultaneously by a $\mathrm{KEVEX} \mathrm{Si}(\mathrm{Li})$ detector and by four wavelength-dispersive (WD) spectrometers. According to the manufacturer's specifications, the $\mathrm{Si}(\mathrm{Li})$ detector consists of an active 3-mm-thick diode covered by a $7-\mu \mathrm{m}$-thick beryllium window and it has an active area of $12.5 \mathrm{~mm}^{2}$. Each WD spectrometer consists of a crystal monochromator and a gas proportional counter (gas-flow-open-type, with a $\mathrm{Be}$ or polypropylene entrance window) with an argon-methane (90:10) mixture as counter gas. Two of the WD spectrometers are separated from the electron column by means of a mylar window, while in the other two, the separation window is made of polypropylene. The $\mathrm{Si}(\mathrm{Li})$ detector and the WD spectrometers are located at directions forming angles of $40^{\circ}$ with respect to the sample surface.

To determine $\mathrm{x}$-ray production cross sections we have to measure very low $\mathrm{x}$-ray intensities and thus it is preferable to use the WD spectrometer rather than the $\mathrm{Si}(\mathrm{Li})$ detector. The reason is that the former has better energy resolution than the latter and therefore peak-to-background ratios obtained from measurements with the WD spectrometer are higher than those recorded with the $\mathrm{Si}(\mathrm{Li})$ detector. Therefore, with the WD spectrometer we obtain net peak intensities with a lower uncertainty, especially when the incident electron energy is close to the ionization threshold. However, the absolute efficiency of the WD spectrometer depends on the incoming

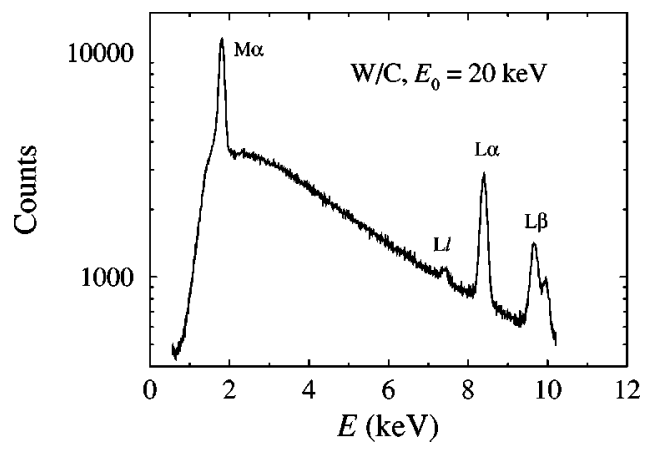

FIG. 1. X-ray spectrum from a 7.5-nm-thick W film deposited on $\mathrm{C}$ obtained with the $\mathrm{Si}(\mathrm{Li})$ detector for an incident electron energy of $20 \mathrm{keV}$.

photon energy in a rather complicated way [19] and it is difficult to estimate. Conversely, the efficiency of the $\mathrm{Si}(\mathrm{Li})$ spectrometer is almost constant in the photon energy of interest in this work (say $8-10 \mathrm{keV}$ ) and it can be determined to good accuracy (see e.g., Ref. [18]). Our approach in the present work consists of combining measurements with both spectrometers: the WD is used to obtain relative $\mathrm{x}$-ray intensities, while the $\mathrm{Si}(\mathrm{Li})$ detector is employed to obtain the absolute value of the $\mathrm{x}$-ray emission cross section for an electron energy of $20 \mathrm{keV}$, which is well above the ionization threshold. In the final stage, the x-ray intensities measured with the WD spectrometer are scaled and converted into absolute $\mathrm{x}$-ray production cross sections by matching the crosssection value determined from the measurement using the $\mathrm{Si}(\mathrm{Li})$ detector.

$\mathrm{X}$-ray measurements were performed on the WD spectrometer using a LiF diffracting crystal for incident electron energies ranging from $10 \mathrm{keV}$ to $30 \mathrm{keV}$ in $1-2 \mathrm{keV}$ steps. The electron-beam current and beam diameter were $40 \mathrm{nA}$ and $20 \mu \mathrm{m}$, respectively. The intensity of characteristics $\mathrm{X}$-rays was counted on the wavelength channel corresponding to the maximum of the characteristic peak and the background was subtracted using linear interpolation of the intensities on channels at both sides of the peak. Counting times of about $100 \mathrm{sec}$ were used for each measured channel. For each sample and beam energy, three measurements were performed at different positions on the sample to test for possible inhomogeneities in the thickness of the active film. Therefore, the standard deviation of the measurements accounts for uncertainties not only due to counting statistics, but also to (minor) inhomogeneities of the active layer. The relative uncertainties of the cross-section values reported below range from $\sim 1 \%$ to $3.8 \%$ (at $1 \sigma$ level).

The $\mathrm{Si}(\mathrm{Li})$ measurements at $20 \mathrm{keV}$ were performed with a beam current of $5 \mathrm{nA}$, a beam diameter of $20 \mu \mathrm{m}$ and an acquisition time of $1000 \mathrm{sec}$. A typical example of an X-ray spectrum obtained with this spectrometer from the $\mathrm{W}$ target bombarded with electrons of $20 \mathrm{keV}$ is displayed in Fig. 1. Usually two spectra were acquired from each sample. For the studied high- $Z$ elements, the characteristic $L$ peaks are well separated and their intensities $N_{L \alpha}$ can be easily obtained either by summing the channel counts directly or by fitting the peak with a Gaussian function, after subtraction of the linearly interpolated background. The statistical uncertainties 
of the resulting $L \alpha$ intensities of interest were less than $1 \%$. The number of incident electrons $N_{\mathrm{e}}$ was evaluated by multiplying the probe current by the "live" acquisition time. To determine the efficiency of the $\mathrm{Si}(\mathrm{Li})$ detector, x-ray spectra were also acquired from a pure graphite substrate and the same instrumental conditions (see below).

The studied samples were thin W, Pt, and Au films deposited on graphite substrates. Au films were produced by resistive evaporation. $\mathrm{W}$ and $\mathrm{Pt}$ films were obtained by sputtering. Graphite was selected as the substrate because of its low atomic number and the associated small electron backscattering.

The thickness of the Au films was controlled by a quartz crystal during evaporation. As the accuracy of the quartz crystal reading was not known, the following calibration procedure was adopted. A number of samples with Au overlayers of different thicknesses were produced, corresponding to various crystal frequency variations $\Delta \nu$. The thicknesses $t$ of the overlayers were determined by Rutherford backscattering spectrometry (RBS) as follows (see e.g., Ref. [20]). The samples were irradiated with a $0.9-\mathrm{MeV} \mathrm{He}^{++}$beam, at normal incidence, in a Tandetron accelerator from a $3-\mathrm{MeV}$ high-voltage engineering [21] at the Ion Implantation Laboratory of the Universidade Federal do Rio Grande do Sul. Ion spectra were detected at $165^{\circ}$, with respect to the incident beam, and were analyzed by means of an analytical method [22] and the RUMP (version 2.0) simulation code [23]. The two methods gave results that agreed to within $1.5 \%$. This procedure allowed the calibration of the quartz crystal for thicknesses larger than about $20 \mathrm{~nm}$, for which the RBS data were reliable enough (the relative uncertainty increases rapidly for decreasing thicknesses). The calibration gave an almost perfect linear dependence of $\Delta \nu$ and $t$ for $t>20 \mathrm{~nm}$, which was also assumed to hold for thinner films. Once the crystal was calibrated, it was utilized in the production of a thin Au overlayer for the x-ray measurements; its thickness was $7.1 \mathrm{~nm}$, small enough to minimize the effect of electron angular and energy straggling.

$\mathrm{W}$ and Pt films were generated by sputtering and their thicknesses were determined from EPMA measurements. This method requires measuring characteristic lines from the overlayer and the substrate at different electron incident energies (for a general description of the thickness determination technique by EPMA; see, e.g., Scott et al. [45]). As the EPMA measurements of carbon are difficult because of the low energy of C $K \alpha$ x rays (see e.g., Ref. [24]), during the sample fabrication runs, twin films of the studied elements were deposited on ultrapure Si targets, which were used for the thickness determination as follows. We measured the ratio of x-ray intensities (the so-called $k$ ratio) of $\mathrm{W} M \alpha, \mathrm{Pt}$ $M \alpha$, and $\mathrm{Si} K \beta$ peaks from the $\mathrm{W} / \mathrm{Si}$ and $\mathrm{Pt} / \mathrm{Si}$ targets to the $\mathrm{x}$-ray intensities of the same peaks from pure $\mathrm{W}, \mathrm{Pt}$, and $\mathrm{Si}$ targets, from $6 \mathrm{keV}$ to $20 \mathrm{keV}$ electron incident energies, in 2 $\mathrm{keV}$ steps. We also measured the Au overlayer on $\mathrm{Si}(\mathrm{Au}$ $M \alpha$ ), which allowed us to compare the EPMA results with the thickness determined by using the quartz crystal. Measured $k$ ratios were analyzed with the help of the X-FILM [25] and LAYERF [26] EPMA analysis codes. These codes estimate the thickness and the elemental concentration of a multilayer

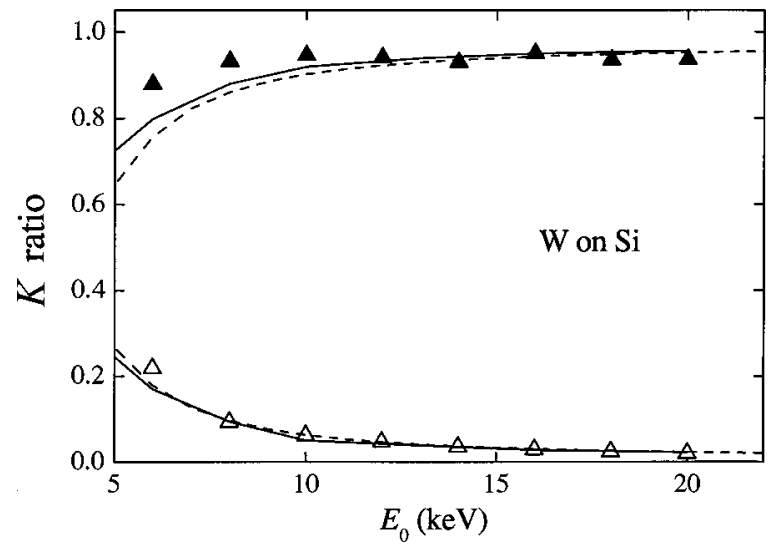

FIG. 2. $K$ ratios for W $M \alpha$ and Si $K \beta$ lines from a 7.5-nm-thick W film deposited on Si substrate, as functions of the incident electron energy. The $k$ ratio was determined with respect to pure $\mathrm{W}$ for the $\mathrm{W} M \alpha$ line and relative to pure Si for the $\mathrm{Si} K \beta$ line. Open triangles are $k$ ratios for the $\mathrm{W} M \alpha$ line; filled triangles are $k$ ratios for the Si $K \beta$ line. Curves are the best predictions of the LAYERF and X-FILM EPMA codes.

target by fitting an analytical model to the experimental $k$ ratios. As an example, Fig. 2 shows the $k$ ratio as a function of the incident electron energy for $\mathrm{W} M \alpha$ and Si $K \beta$ lines from a W/Si sample, as well as the best predictions of the LAYERF and X-FILM codes. Thicknesses obtained using the different methods are summarized in Table I. We can see that for the $\mathrm{Au}$ sample, the result obtained from the calibrated quartz crystal is in good agreement with the result obtained from the two EPMA methods, as their results are also seen to agree satisfactorily. For each sample, the adopted thickness was set equal to the average of the values obtained from the available methods.

\section{DATA CORRECTION}

Although the active films are very thin (see Table I), incident electrons do suffer some scattering and lose energy within the film. Therefore, the "effective" path length of electrons is somewhat larger than the film thickness and the effective energy is slightly smaller than the energy of incidence. Furthermore, electrons backscattered from the substrate may also produce ionization within the active film, which causes an enhancement of emitted characteristic $\mathrm{x}$ rays, and also bremsstrahlung photons.

TABLE I. Film thicknesses and associated uncertainties obtained by EPMA using the codes LAYERF and X-FILM and by the quartz crystal, calibrated by RBS.

\begin{tabular}{|c|c|c|c|}
\hline \multirow[t]{3}{*}{ Element } & \multicolumn{3}{|c|}{ Thickness (nm) } \\
\hline & EPMA & EPMA & Quartz crystal \\
\hline & LAYERF & X-FILM & \\
\hline W & $7.5 \pm 0.2$ & $7.5 \pm 0.3$ & \\
\hline $\mathrm{Pt}$ & $12.0 \pm 0.3$ & $11.7 \pm 0.6$ & \\
\hline $\mathrm{Au}$ & $7.0 \pm 0.2$ & $7.2 \pm 0.1$ & $7.1 \pm 0.2$ \\
\hline
\end{tabular}



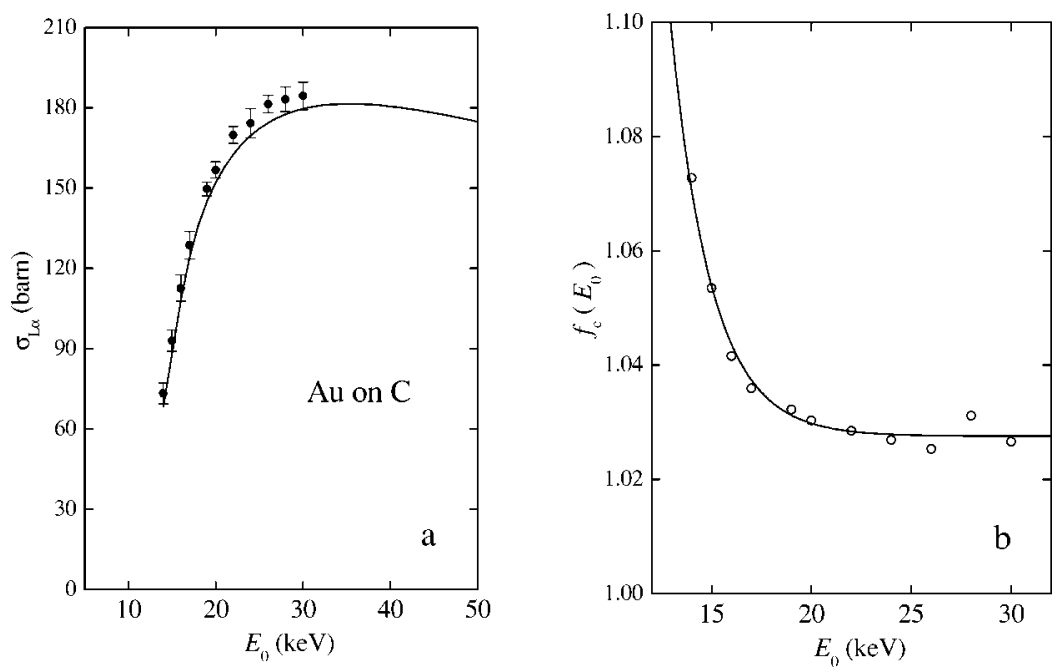

FIG. 3. Theoretical $L \alpha$ x-ray production cross sections vs electron incident energy for $\mathrm{Au}$, calculated for a thin self-supporting film by using ionization cross sections calculated from the DWBA and atomic relaxation data from the EADL (solid line) and derived from the Monte Carlo simulation of emission from a 7.1-nm-thick $\mathrm{Au} / \mathrm{C}$ sample using identical ionization cross sections and relaxation data (symbols) (a) and correction factors $f_{\mathrm{c}}$ vs incident electron energy for the same composite sample (b). The solid line in the right panel is the curve given by Eq. (4), with fitted parameter values.

To account for these effects, we have carried out MC simulations of $\mathrm{X}$-ray spectra emitted from samples with the two-layer structure used in our measurements for incident electron beams with energies between 10 and $30 \mathrm{keV}$. The simulations were performed by using a dedicated MC subroutine set named PEN-XR [27], which is based on the latest version of the general-purpose subroutine package PENELOPE [28]. PEN-XR implements the most accurate interaction models available to date. Bremsstrahlung emission is described by the means of differential cross sections (DCS), differential in the energy and direction of the emitted photon, obtained by combining scaled DCS (i.e., photon energy spectra) interpolated from Seltzer and Berger's database [29] and angular distributions obtained from an accurate parametrization of the "shape functions" tabulated by Kissel et al. [30]. The ionization of inner shells by electron impact is described by using theoretical total ionization cross sections, calculated by means of a relativistic DWBA code $[2,31]$. The relaxation of the produced ions is simulated using transition probabilities from the evaluated atomic data library (EADL) [32].

From the simulated $\mathrm{x}$-ray spectra, $L \alpha$ x-ray intensities $I_{L \alpha, \mathrm{MC}}$ were obtained in absolute units (i.e., as the number of photons emitted per unit solid angle per incident bombarding electron) and subsequently converted into $L \alpha$ x-ray production cross sections by using the equation

$$
\sigma_{L \alpha, \mathrm{MC}}=\frac{1}{\mathcal{N} t} I_{L \alpha, \mathrm{MC}}
$$

where $\mathcal{N}$ is the number of atoms per unit volume and $t$ is the simulated target thickness. The DWBA ionization cross sections $\sigma_{L i}$ used in the MC simulations were in turn converted to $L \alpha$ x-ray production cross sections $\sigma_{L \alpha}$ using the formula (see e.g., Ref. [9])

$$
\begin{aligned}
\sigma_{L \alpha, \text { th }}= & \frac{\Gamma_{M 4,5-L 3}}{\Gamma_{\text {Total }-L_{3}}} \omega_{L_{3}}\left[\sigma_{L_{3}}+f_{23} \sigma_{L_{2}}+\left(f_{13}\right.\right. \\
& \left.\left.+f_{12} f_{23}\right) \quad \sigma_{L_{1}}\right]
\end{aligned}
$$

where $\Gamma_{M_{4,5}-L_{3}}$ and $\Gamma_{\text {Total }-L_{3}}$ are the x-ray emission rates for $L \alpha\left(M_{4,5}-L_{3}\right.$ transition) and total ( $M, N, O-L_{3}$ transitions) $\mathrm{x}$ rays, respectively, $\omega_{L_{3}}$ is the fluorescence yield for the $L_{3}$ shell, and $f_{i j}$ are the Coster-Kronig transition probabilities. These parameters were obtained by combining the corresponding transition probabilities extracted from the EADL.

Figure 3(a) compares theoretical $L \alpha$ x-ray production cross sections $\sigma_{L \alpha, \text { th }}$, calculated within the DWBA and using relaxation data obtained from the EADL, with the production cross section inferred from the MC simulation, $\sigma_{L \alpha, \mathrm{MC}}$, of emission from a 7.1-nm-thick Au/C sample. The error bars associated with the $\mathrm{MC}$ results represent statistical uncertainties (three standard deviations). Notice that the DWBA calculated cross section is that used in the simulation and, therefore, the two data sets displayed in Fig. 3(a) would coincide in the case of a self-supporting, infinitely thin film. The objective of this figure is to reveal the combined effect of finite film thickness and backscattering from the substrate, which can be expressed by means of a correction factor

$$
f_{\mathrm{c}}=\frac{\sigma_{L \alpha, \mathrm{MC}}}{\sigma_{L \alpha, \mathrm{th}}},
$$

where $\sigma_{L \alpha, \mathrm{MC}}$ and $\sigma_{L \alpha \text {,th }}$ are given by Eqs. (1) and (2), respectively. This correction is expected to be fairly insensitive to the details of the adopted ionization cross section. As seen in Fig. 3(b), and also for other overlayers and substrates used in this work, the largest correction is of the order of $10 \%$.

Correction factors $f_{\mathrm{c}}$ calculated in this way have been used to transform measured $\mathrm{x}$-ray intensities into relative $\mathrm{X}$-ray production cross sections, i.e., $\mathrm{x}$-ray intensities that would result from a bare active film with neither electron scattering nor energy loss. As illustrated in Fig. 3(b), the correction factor increases as the electron incident energy $E_{0}$ approaches the ionization threshold. In order to minimize the effect of statistical uncertainties, the correction factor has been approximated by the following analytical expression:

$$
f_{\mathrm{c}}\left(E_{0}\right)=A+B \exp \left(-C E_{0}\right)
$$




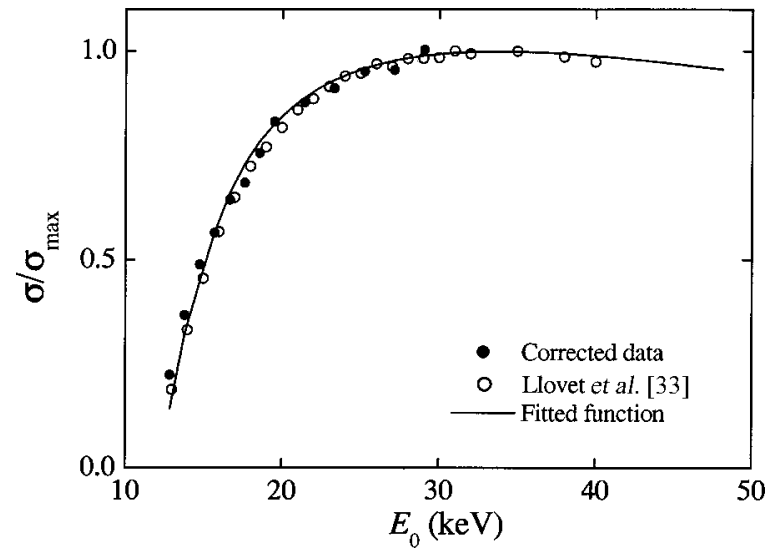

FIG. 4. Relative $L \alpha$ x-ray production cross sections for Au vs incident electron energy. Full circles are the results of the present measurements using $\mathrm{Au} / \mathrm{C}$ samples. Open circles represent measurements by Llovet et al. [33] using self-supporting thin film samples. The continuous line is the result of a fit with the analytical form given by Eq. (8).

with parameters determined by fitting the calculated crosssection ratios. In Fig. 4, we compare our corrected measurements of relative $L \alpha$ x-ray production cross sections of $\mathrm{Au}$ with the measurements of Llovet et al. [33], which were obtained by using a 3.9-nm-thick self-supporting film. The agreement between these two independent experiments corroborates the correctness of the correction procedure adopted here.

\section{DATA ANALYSIS}

For an homogeneous film of thickness $t$ and normal electron-beam incidence, the absolute x-ray production cross section $\sigma_{L \alpha}$ can be expressed as (see e.g., Ref. [9])

$$
\sigma_{L \alpha}\left(E_{0}\right)=\frac{4 \pi}{\mathcal{N} t N_{\mathrm{e}} f_{\mathrm{c}}\left(E_{0}\right) \epsilon\left(E_{\mathrm{ph}}\right) \Delta \Omega} N_{L \alpha}\left(E_{0}\right),
$$

where $N_{L \alpha}$ is the intensity of the characteristic $L \alpha$ peak, $E_{0}$ is the incident electron energy, $E_{\mathrm{ph}}$ is the emitted photon energy, $\mathcal{N}$ is the number of atoms per unit volume, $t$ is the target thickness, $N_{\mathrm{e}}$ is the number of incident electrons, $\epsilon\left(E_{\mathrm{ph}}\right)$ and $\Delta \Omega$ are the intrinsic efficiency and solid angle subtended by the spectrometer, and $f_{\mathrm{c}}\left(E_{0}\right)$ is the correction factor defined above, which accounts for electron-transport corrections. Notice that for an ideal, infinitely thin selfsupporting film, we would have $f_{\mathrm{c}}\left(E_{0}\right)=1$.

As mentioned above, it is difficult to determine the efficiency and the solid angle subtended by a WD spectrometer. To avoid this difficulty, we have derived absolute crosssection values from measurements with the $\mathrm{Si}(\mathrm{Li})$ detector, whose efficiency and solid angle can be determined more accurately, by using two alternative methods. The first is based on Eq. (5) and requires determining all the parameters in this equation. The second method, which is described in the Appendix, relies on calculated and experimental bremsstrahlung cross sections [5]. Evidently, uncertainties in the adopted bremsstrahlung cross sections will spread to the re-

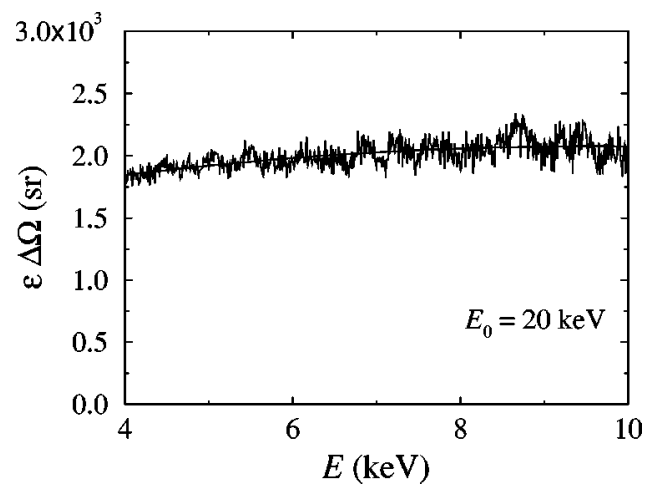

FIG. 5. Estimated absolute detector efficiency of the $\mathrm{Si}(\mathrm{Li})$ detector as a function of emitted photon energy.

sulting x-ray production cross sections. Absolute cross sections obtained from these two scaling methods are consistent with the estimated uncertainties.

Although in the photon energy range of interest, say 8-10 $\mathrm{keV}$, the absolute efficiency of a $\mathrm{Si}(\mathrm{Li})$ spectrometer can be determined by relatively simple means (see e.g., Ref. [18]), the solid angle of the detector was not known with sufficient accuracy. To overcome this difficulty, we have estimated the detector efficiency with the help of MC simulation as follows. Let us consider the number of photons $N_{\text {exp }}(E)$ detected per unit energy interval and unit solid angle per incident electron from a thick solid target, irradiated with an electron beam of energy $E_{0}$. This can be expressed as

$$
N_{\text {exp }}(E)=\frac{N_{\mathrm{ch}}(E)}{N_{\mathrm{e}} \epsilon(E) \Delta \Omega \Delta E},
$$

where $N_{\mathrm{ch}}(E)$ is the number of counts in a particular photon energy channel of width $\Delta E$ centered at $E$. If we replace $N_{\text {exp }}(E)$ by the result from a MC simulation expressed in absolute units $\left(N_{\mathrm{MC}}\right)$, then $\epsilon \Delta \Omega$ can be calculated as

$$
\epsilon(E) \Delta \Omega=\frac{N_{\mathrm{ch}}(E)}{N_{\mathrm{e}} N_{\mathrm{MC}}(E) \Delta E} .
$$

In a previous work, we have shown that simulated thicktarget bremsstrahlung spectra from pure carbon targets, obtained with the aid of our MC tool PEN-XR, are in very good agreement with absolute X-ray spectra measured with a $\mathrm{Si}(\mathrm{Li})$ detector [27]. Therefore, we have obtained the detector efficiency from Eq. (7) by combining our measured thicktarget bremsstrahlung spectra on graphite targets with MC simulations. As the detector efficiency is nearly constant on a wide energy interval (see Fig. 5), we have adopted the average value of $\epsilon \Delta \Omega$ in a photon energy region around the energies of interest, i.e., W $L \alpha\left(E_{\mathrm{ph}}=8.396 \mathrm{keV}\right)$, Pt $L \alpha$ $\left(E_{\mathrm{ph}}=9.441 \mathrm{keV}\right)$, and Au $L \alpha\left(E_{\mathrm{ph}}=9.712 \mathrm{keV}\right)$.

Cross-section measurements are affected by relative uncertainties, which arise mainly from counting statistics, background subtraction, sample nonuniformity, and instrumental drift during measurements; they were, on an average, $1.9 \%$ for $\mathrm{W}, 2.5 \%$ for $\mathrm{Pt}$, and $2.4 \%$ for Au. Relative uncertainties only affect the shape of the cross-section curve. The conver- 
sion (scaling) from relative cross sections to absolute cross sections introduces additional uncertainties of a systematic nature, which are the same for all measured energies. These are estimated to be $\sim 8 \%$ and originate from uncertainties in the determination of film thicknesses $(4.5 \%$ for $\mathrm{W}, 5.3 \%$ for $\mathrm{Pt}$, and $3.7 \%$ for $\mathrm{Au}$ ), detection efficiency (6\%), number of incident electrons $(2 \%)$, and the statistical uncertainties of peak measurements with the $\mathrm{Si}(\mathrm{Li})(1 \%)$. With the alternative method described in the Appendix, systematic uncertainties are estimated to be $\sim 10.5 \%$ and arise from the uncertainty of the adopted (calculated) bremsstrahlung cross sections (10\%) [30] and from the statistical uncertainties of measured peak-to-background ratios with the $\mathrm{Si}(\mathrm{Li})(3.2 \%$ for $\mathrm{W}, 1.9 \%$ for $\mathrm{Pt}$, and $2.2 \%$ for $\mathrm{Au}$ ).

The $\mathrm{x}$-ray production cross-section values reported in this study are calculated as the mean of the cross sections obtained from Eqs. (5) and (A3) (see the Appendix). These have been found to agree to within $\sim 7 \%$. This agreement provides a consistency check of the conversion procedure. The absolute uncertainty of the reported cross sections, obtained by combining relative and systematic uncertainties in quadrature, is estimated to be $\sim 11 \%$.

\section{RESULTS AND DISCUSSION}

In what follows, we compare our results with the predictions of various theoretical ionization cross-section models. We consider the classical formula of Gryzinski [34] and the semiempirical formula of Worthington-Tomlin [35], which are widely used in EPMA. We also consider results from recent relativistic DWBA calculations by Segui et al. [2]. These calculations go beyond the better known plane-wave Born approximation by accounting for (1) the distortion of the projectile wave functions by the field of the target atom and (2) electron exchange effect.

The $L$-shell ionization cross sections obtained from these models/calculations have been converted into $L \alpha$ x-ray production cross sections by using Eq. (2). The x-ray emission rates, fluorescence yields, and Coster-Kronig coefficients have been taken from different available bibliographic sources [32,36-43]. As already pointed out, these relaxation data are affected by sizable uncertainties and, for a given set of theoretical $L$-shell ionization cross sections, the $L \alpha$ x-ray production cross sections obtained with relaxation data from the various available sources are found to lie within an "uncertainty band" whose width is $\sim 8 \%$ for Pt, $\sim 17 \%$ for W, and $\sim 8 \%$ for $\mathrm{Au}$. Notice that if we had tried to infer $L$-shell ionization cross sections from measured $L \alpha$ x-ray intensities, these uncertainties would have added to those arising from the measurement. In order to facilitate the comparisons, we have adopted the theoretical fractional emission rates given by Scofield [36] and the experimental fluorescence yields, and Coster-Kronig coefficients given by Werner and Jitschin [39], which are summarized in Table II.

Cross sections for $L \alpha$ x-ray production of $\mathrm{Au}, \mathrm{W}$, and $\mathrm{Pt}$ are listed in Table III. The data are plotted in Fig. 6 together with representative absolute uncertainties that, as mentioned above, are of the order of $11 \%$. Notice, however, that the shape of the cross-section curve is much more accurate
TABLE II. X-ray emission rates, fluorescence yields, and Coster-Kronig transition probabilities used in this work, taken from Scofield [36] and Werner and Jitschin [39].

\begin{tabular}{cccccc}
\hline \hline Element & $\Gamma_{M_{4,5}-L_{3}} / \Gamma_{\text {Total }-L_{3}}$ & $\omega_{L_{3}}$ & $f_{12}$ & $f_{13}$ & $f_{23}$ \\
\hline $\mathrm{W}$ & 0.80 & 0.245 & 0.102 & 0.325 & 0.106 \\
$\mathrm{Pt}$ & 0.79 & 0.294 & 0.066 & 0.562 & 0.104 \\
$\mathrm{Au}$ & 0.78 & 0.307 & 0.047 & 0.582 & 0.101 \\
\hline \hline
\end{tabular}

$(\sim 3 \%)$, since it is only affected by relative uncertainties. This is clearly seen from the smoothness of the measured cross section vs energy plots (Fig. 6). Our measurements are also compared with experimental data from other authors in Fig. 6. Some of the experimental results found in the literature were given as subshell ionization cross sections $[7,10,11]$, therefore these have been converted into $L \alpha$ x-ray production cross sections by using the relaxation parameters adopted by the authors to do the reverse transformation. However, in some cases $[7,10]$, the authors did not provide experimental information on all the required subshells. In these cases, we have assumed subshell cross-section ratios identical to those obtained from DWBA calculations at 20 $\mathrm{keV}$. Namely, for $\mathrm{Au}, \sigma_{L 3} / \sigma_{L 1}=0.356$ and $\sigma_{L 3} / \sigma_{L 2}$ $=0.246$.

In the case of $\mathrm{Au}$ [Fig. 6(c)], our results agree quite well with the experiments of Shima et al. [9], Davis et al. [8], and Schneider et al. [10]. The latter measurements, however, are smaller than ours and the difference increases with incident electron energies, whereas the measurements of Davis et al. [8] at $20 \mathrm{keV}$ are somewhat higher than ours. The measurements of Salem and Moreland [7] lie systematically (about $60 \%$ ) below our results. For W [Fig. 6(a)], the measurements of Chang [11] are about $40 \%$ lower than our experimental results. For Pt [Fig. 6(b)], no experimental data were found in the literature. As regards the comparison with the theoret-

TABLE III. Measured $L \alpha$ x-ray production cross sections for W, $\mathrm{Pt}$, and $\mathrm{Au}$. The absolute uncertainties are about $11 \%$.

\begin{tabular}{cccc}
\hline \hline & W & Pt & Au \\
$\begin{array}{c}\text { Energy } \\
(\mathrm{keV})\end{array}$ & $\begin{array}{c}\text { Cross section } \\
(\mathrm{b})\end{array}$ & $\begin{array}{c}\text { Cross section } \\
(\mathrm{b})\end{array}$ & $\begin{array}{c}\text { Cross section } \\
(\mathrm{b})\end{array}$ \\
\hline 12 & 68 & & \\
13 & 91 & 39 & 52 \\
14 & 108 & 69 & 75 \\
15 & 125 & 89 & 93 \\
16 & 137 & 105 & 106 \\
17 & 147 & 120 & 118 \\
18 & 155 & 128 & 124 \\
19 & 162 & 138 & 136 \\
20 & 168 & 146 & 148 \\
22 & 176 & 155 & 155 \\
24 & 181 & 161 & 160 \\
26 & 186 & 163 & 166 \\
28 & 186 & 165 & 167 \\
30 & 187 & 167 & 174 \\
\hline \hline
\end{tabular}



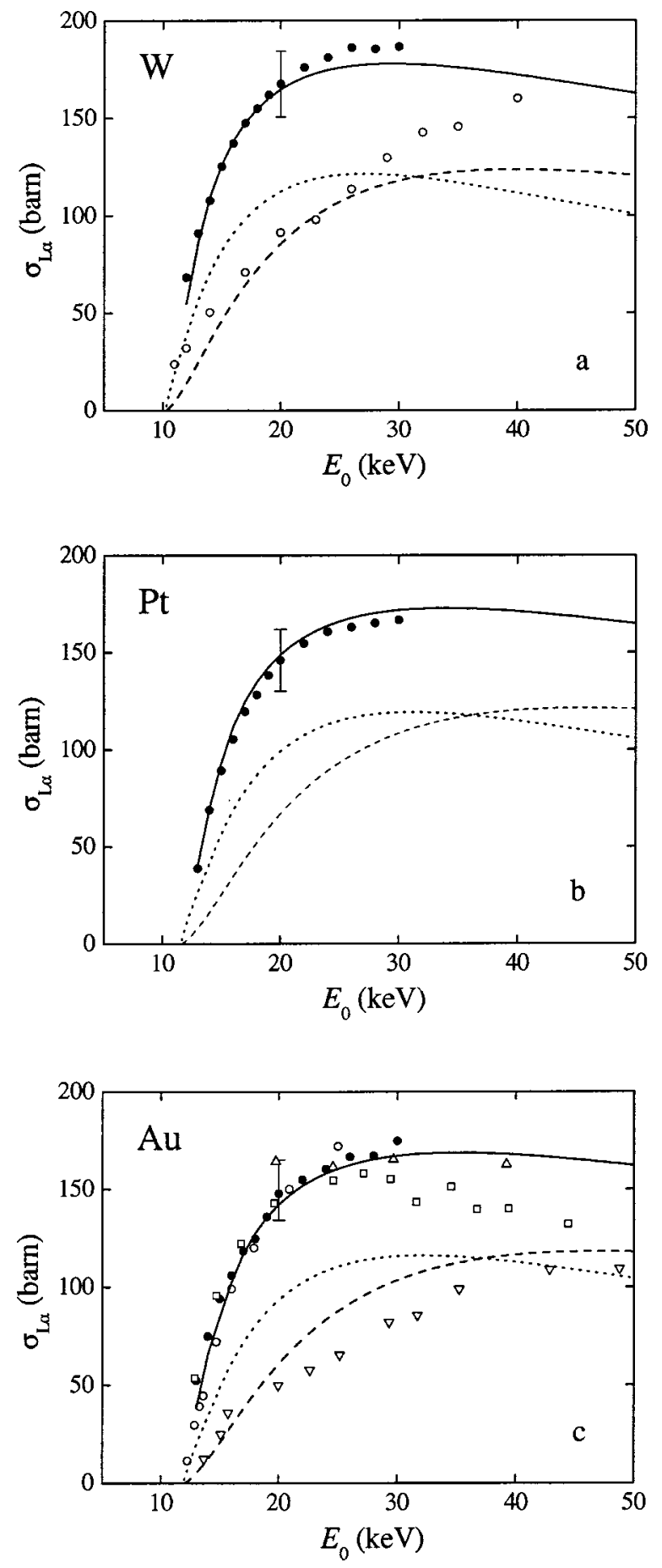

FIG. 6. $L \alpha$ x-ray production cross section vs incident electron energy for $\mathrm{W}(\mathrm{a}), \mathrm{Pt}(\mathrm{b})$, and $\mathrm{Au}$ (c). The curves have been obtained by means of Eq. (2) using ionization cross sections calculated from different formulas and approximations. The dotted curves are from the Gryzinski formula [34]; the dashed curves represent results from the Worthington-Tomlin [35] formula; the continuous curves were obtained from distorted-wave ionization cross sections calculated by Segui et al. [2]. Full circles represent the results from the present measurements. Open symbols are experimental data from by Chang [11] (circles), Shima et al. [9] (circles), Schneider et al. [10] (squares), Davis et al. [8] (triangles), and Salem and Moreland [7] (inverted triangles).

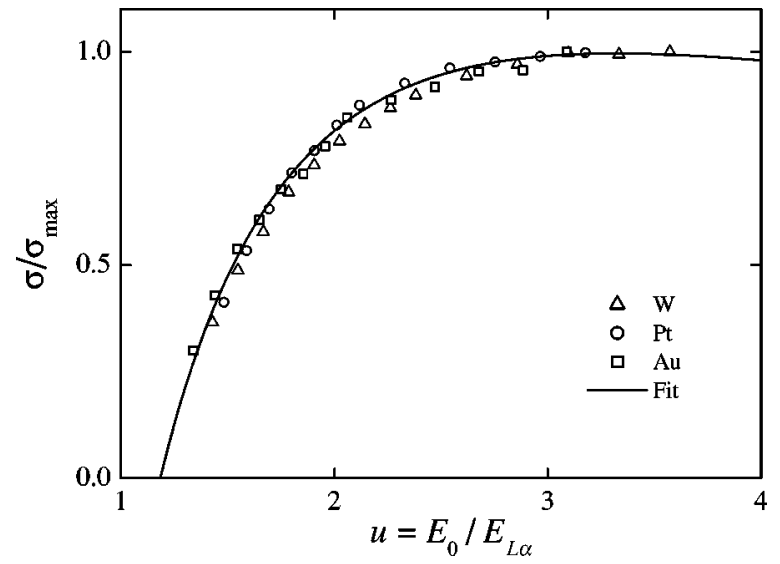

FIG. 7. Relative cross section as a function of the overvoltage $u$. Symbols denote measured data for the indicated elements. The continuous curve is the function given by Eq. (8), with the parameters indicated in the text.

ical results, for the three measured elements, the DWBA calculations are in excellent agreement with our experimental data, while results from the Gryzinski and WorthingtonTomlin formulas are systematically lower than our data.

Figure 7 shows our cross sections for the various elements normalized to their corresponding maxima $\sigma_{\max }$, as functions of the overvoltage $u=E_{0} / E_{L \alpha}$,

$$
\sigma_{L \alpha}(u) / \sigma_{\max }=\frac{A_{1}}{\left(u+A_{2}\right)} \frac{1}{u^{A_{3}}} \ln \left(u+A_{4}\right),
$$

where $A_{1}=3.950, A_{2}=0.70, A_{3}=0.10$, and $A_{4}=0.184$ are parameters, which have been determined from a numerical fit to all the measured data. It is seen that, within the experimental uncertainties, this function describes all the measurements reasonably well for the three considered elements. On the other hand, the small spread of the experimental data about the fitted curve does confirm the relative accuracy of our measurements.

In conclusion, we have reported measurements of $L \alpha$ $\mathrm{x}$-ray production cross sections for the elements $\mathrm{W}, \mathrm{Pt}$, and $\mathrm{Au}$, from threshold up to $30 \mathrm{keV}$. The adopted experimental procedure and evaluation methods allowed us to reduce the relative and absolute uncertainties to about $3 \%$ and $11 \%$, respectively. The DWBA-based calculations of Segui et al. [2], combined with x-ray emission rates from Scofield [36], fluorescence yields, and Coster-Kronig coefficients from Werner and Jitschin [39], have been found to be in excellent agreement with our measurements.

\section{ACKNOWLEDGMENTS}

We would like to thank Dr. J. M. Fernández-Varea and Dr. S. Segui for their help with the theoretical calculations, Dr. C. Merlet for allowing us to use the X-FILM software, and Mr. P.E.G.C. Silva Jr. for his technical assistance during the sample preparation. C.S.C. wishes to acknowledge financial support from CNPq-Brasil (Project No. 200784/00-5). 


\section{APPENDIX}

In this Appendix, we describe an alternative method to that presented in Sec. IV to derive the absolute x-ray production cross section from the measured data. In the case of a homogeneous film of thickness $t$ and an electron beam at normal incidence, the double-differential cross section (differential in energy and direction of the emitted photon) for emission of bremsstrahlung photons of energy $E$ can be expressed as (see e.g., Ref. [44])

$$
\frac{d \sigma_{\mathrm{b}}}{d \Omega d E}=\frac{N_{\mathrm{b}}}{\mathcal{N} t N_{\mathrm{e}} \epsilon(E) \Delta \Omega \Delta E},
$$

where, as before, $N_{\mathrm{b}}$ is the number of detected bremsstrahlung photons in an energy channel of width $\Delta E$ centered at the energy $E, \mathcal{N}$ is the number of atoms per unit volume, $N_{\mathrm{e}}$ is the number of incident electrons, and $\epsilon$ and $\Delta \Omega$ are the intrinsic efficiency and solid angle subtended by the $\mathrm{x}$-ray detector. Taking the ratio of the cross section for $\mathrm{x}$-ray emission [Eq. (5)], to the cross section for bremsstrahlung emission [Eq. (A1)], we obtain

$$
\frac{\sigma_{L \alpha}}{d \sigma_{\mathrm{b}} /(d \Omega d E)}=\frac{N_{L \alpha}}{N_{\mathrm{b}}} \frac{\epsilon(E)}{f_{\mathrm{c}}\left(E_{0}\right) \epsilon\left(E_{\mathrm{ph}}\right)} 4 \pi \Delta E .
$$

Assuming that the energy of the characteristic peak $E_{\mathrm{ph}}$ is close to the energy $E$ where the bremsstrahlung background is measured, $\epsilon(E) \sim \epsilon\left(E_{\mathrm{ph}}\right)$ and we have

$$
\sigma_{L \alpha}=4 \pi \frac{N_{L \alpha}}{N_{\mathrm{b}}} \frac{1}{f_{\mathrm{c}}\left(E_{0}\right)} \frac{d \sigma_{\mathrm{b}}}{d \Omega d E} \Delta E .
$$

[1] J.H. Scofield, Phys. Rev. A 18, 963 (1978).

[2] S. Segui, E. Acosta, and F. Salvat (unpublished).

[3] A. Langenberg, F.J. de Heer, and J. van Eck, J. Phys. B 8, 2079 (1975).

[4] R. Hippler, H. Klar, K. Saeed, I. McGregor, A.J. Duncan, and H. Kleinpoppen, J. Phys. B 16, L617 (1983).

[5] C.A. Quarles and M. Semaan, Phys. Rev. A 26, 3147 (1982).

[6] R. Hippler, I. McGregor, M. Aydinol, and H. Kleinpoppen, Phys. Rev. A 23, 1730 (1981).

[7] S.I. Salem and L.D. Moreland, Phys. Lett. 37A, 161 (1971).

[8] D.V. Davis, V.D. Mistry, and C.A. Quarles, Phys. Lett. 38A, 169 (1972).

[9] K. Shima, T. Nakagawa, K. Umetani, and T. Mikumo, Phys. Rev. A 24, 72 (1981).

[10] H. Schneider, I. Tobehn, F. Ebel, and R. Hippler, Phys. Rev. Lett. 71, 2707 (1993).

[11] C.N. Chang, Phys. Rev. A 19, 1930 (1979).

[12] J.J. Vrakking and F. Meyer, Phys. Rev. A 9, 1932 (1974).

[13] J.H. Paterson, J.N. Chapman, W.A.P. Nicholson, and J.M. Titchmarsh, J. Microsc. 154, 1 (1988).

[14] D.C. Joy, J. Microsc. 191, 74 (1998).

[15] C.J. Powell, Rev. Mod. Phys. 48, 33 (1976)

[16] C.J. Powell, in Microbeam Analysis, edited by J.R. Michael
This equality holds for thin self-supporting films (i.e., without substrate), but cannot be directly applied to the kind of samples used in the present study, films on substrates. To avoid this difficulty, we have simulated x-ray spectra emitted from samples with the geometrical structure used in our measurements and we have replaced $d \sigma_{\mathrm{b}} / d \Omega d E$ in Eq. (A3) by the estimate $d \sigma_{\mathrm{b}, \mathrm{MC}} / d \Omega d E$ obtained from the MC simulation,

$$
\frac{d \sigma_{\mathrm{b}, \mathrm{MC}}}{d \Omega d E}=\frac{1}{\mathcal{N} t} \frac{N_{\mathrm{b}, \mathrm{MC}}}{N_{\mathrm{e}} \Delta \Omega \Delta E}=\frac{1}{\mathcal{N} t} I_{\mathrm{b}, \mathrm{MC}},
$$

where $I_{\mathrm{b}, \mathrm{MC}}$ is the simulated bremsstrahlung absolute intensity (i.e., number of photons emitted with energy $E$ in the direction of the detector per unit energy interval and unit solid angle per incident electron) and $t$ is the thickness of the active film, which was set equal to the average of measured values given in Table I. Combining Eqs. (A4) and (A3), we finally obtain the sought formula

$$
\sigma_{L \alpha}=4 \pi \frac{N_{L \alpha}}{N_{\mathrm{b}}} \frac{1}{f_{\mathrm{c}}\left(E_{0}\right)} \frac{1}{\mathcal{N} t} I_{\mathrm{b}, \mathrm{MC}}(E) \Delta E .
$$

With this method the effects of electron scattering and energy loss within the film and backscattering in the substrate on the emitted bremsstrahlung spectrum are accounted for in a consistent way. and P. Ingram (San Francisco Press, San Francisco, 1990).

[17] C.J. Powell, in Electron Impact Ionization, edited by T.D. Mark and D.H. Dunn (Springer-Verlag, Berlin, 1985), Chap. 6.

[18] X. Llovet, C. Merlet, and F. Salvat, J. Phys. B 33, 3761 (2000).

[19] S. Reusch, H. Genz, W. Löw, and A. Richter, Z. Phys. D: At., Mol. Clusters 3, 379 (1986).

[20] W.K. Chu, J.W. Mayer, and M.A. Nicolet, Backscattering Spectrometry (Academic Press, New York, 1978).

[21] M. Friedrich, W. Bürger, D. Henke, and S. Turuc, Nucl. Instrum. Methods Phys. Res. A 382, 357 (1996).

[22] C.S. Campos, E. Coleoni, J. Trincavelli, J. Kaschny, R. Hubbler, M.R.F. Soares, and M.A.Z. Vasconcellos, X-Ray Spectrom. 30, 253 (2001).

[23] L.R. Doolittle, Ph.D thesis, Cornell University, Ithaca, 1987.

[24] G.F. Bastin and H.J.M. Heijligers, Quantitative Electron Probe Microanalysis of Carbon in Binary Carbides (University of Technology, Eindhoven, The Netherlands, 1985).

[25] C. Merlet, in Proceedings of the 29th Annual Conference of the Microbeam Analysis Society, edited by E.S. Etz. (VHC Publishers, New York, 1995), p. 203.

[26] J.L. Pouchou and F. Pichoir, Scanning 12, 212 (1990).

[27] E. Acosta, X. Llovet, and F. Salvat, Appl. Phys. Lett. 80, 2328 (2002).

[28] F. Salvat, J.M. Fernández-Varea, E. Acosta, and J. Sempau, Computer code PENELOPE (OECD/NEA Data Bank, Issy-lesMoulineaux, France, 2001). 
[29] S.M. Seltzer and M.J. Berger, Nucl. Instrum. Methods Phys. Res. B 12, 95 (1985).

[30] L. Kissel, C.A. Quarles, and R.H. Pratt, At. Data Nucl. Data Tables 28, 381 (1983).

[31] S. Segui, Ph.D thesis, University of Cordoba, Argentina, 2000.

[32] S.T. Perkins, D.E. Cullen, M.H. Chen, J.H. Hubbell, J. Rathkopf, and J. Scofield, Lawrence Livermore National Laboratory, Report No. UCRL-50400, 1991 (unpublished).

[33] X. Llovet, C. Merlet, J.M. Fernández-Varea, and F. Salvat, Mikrochim. Acta 132, 163 (2000).

[34] M. Gryzinski, Phys. Rev. 138, 336 (1965).

[35] C.R. Worthington and S.G. Tomlin, Proc. Phys. Soc., London, Sect. A 69, 401 (1956).

[36] J.H. Scofield, Phys. Rev. 179, 9 (1969).
[37] M.O. Krause, J. Phys. Chem. Ref. Data 8, 307 (1979).

[38] M.H. Chen, B. Crasemann, and H. Mark, Phys. Rev. A 24, 1845 (1981).

[39] U. Werner and W. Jitschin, Phys. Rev. A 38, 4009 (1988).

[40] J.Q. Xu, Phys. Rev. A 43, 4771 (1991).

[41] S. Puri, D. Mehta, B. Chand, N. Singh, and P. Trehan, X-Ray Spectrom. 22, 358 (1993).

[42] M. Şahin, L. Demir, Ö. Sögüut, M. Ertuğrul, and O. İçelli, J. Phys. B 33, 93 (2000).

[43] E. Öz, N. Ekinci, Y. Özdemir, M. Ertuğrul, Y. Şahin, and H. Erdoğan, J. Phys. B 34, 631 (2001).

[44] C.A. Quarles and D.B. Heroy, Phys. Rev. A 24, 48 (1981).

[45] V.D. Scott, G. Love, and S.G.B. Reed, Quantitative Electronprobe Microanalysis (Harwood, New York, 1995). 